\title{
MicroRNA-22 inhibits the proliferation and migration, and increases the cisplatin sensitivity, of osteosarcoma cells
}

\author{
XIANG ZHOU ${ }^{1}$, DIMPLE NATINO ${ }^{2}, \mathrm{XU}_{\mathrm{ZHAI}^{3}}$, $\mathrm{ZHONGYANG} \mathrm{GAO}^{1}$ and XIJING HE ${ }^{1}$ \\ ${ }^{1}$ Department of Orthopedics, Second Affiliated Hospital of Xi'an Jiaotong University, Xi'an, Shaanxi 710004, P.R. China; \\ ${ }^{2}$ Department of Biomedical Sciences, Florida State University College of Medicine, Tallahassee, FL 32304, USA; \\ ${ }^{3}$ Emergency Department, Second Affiliated Hospital of Xi'an Jiaotong University, Xi'an, Shaanxi 710004, P.R. China
}

Received August 16, 2017; Accepted January 3, 2018

DOI: $10.3892 / \mathrm{mmr} .2018 .8790$

\begin{abstract}
Osteosarcoma (OS) is the major type of primary bone tumor and is associated with a poor prognosis due to chemotherapy resistance. Accumulating evidence indicates that microRNAs (miRNAs/miRs) may influence the tumor progression of OS and cell sensitivity to chemotherapy. In the present study, a total of 7 patients with OS and 7 healthy volunteers were recruited. Reverse transcription-quantitative polymerase chain reaction and ELISA were performed to determine the expression of miRNAs and mRNAs in the serum of participants. Furthermore, the biological function of miR-22 and S100A11 was examined in MG-63 cells using Cell Counting Kit-8 assays, Transwell migration assays and western blot analysis to determine the effects on cell proliferation, migration and protein expression, respectively, while MG-63 cell sensitivity to cisplatin was assessed by measuring cell viability following cisplatin treatment and calculating the half maximal inhibitory concentration $\left(\mathrm{IC}_{50}\right)$. Additionally, the association between miR-22 and S100 calcium-binding protein A11 (S100A11) was validated using a luciferase reporter assay. The results demonstrated that miR-22 expression was significantly reduced in patients with OS and the MG-63 OS cell line, compared with healthy volunteers and the normal osteoblast hFOB 1.19 cell line, respectively, while the expression of S100A11 was negatively associated with miR-22 levels in the MG-63 cell line. Furthermore, overexpression of miR-22 inhibited the proliferation and migratory ability of MG-63 cells, and increased the sensitivity of MG-63 cells to cisplatin treatment; however, overexpression of S100A11 partially attenuated the alterations in proliferation, migratory ability and chemosensitivity that were induced by miR-22 overexpression. In addition, it was confirmed that S100A11
\end{abstract}

Correspondence to: Professor Xijing He, Department of Orthopedics, Second Affiliated Hospital of Xi'an Jiaotong University, 157 Xiwu Road, Xi'an, Shaanxi 710004, P.R. China E-mail: xijing_h@vip.tom.com

Key words: osteosarcoma, chemotherapy, microRNA, S100A11, proliferation, migration, cisplatin is a direct target gene of miR-22 in MG-63 cells. In conclusion, to the best of our knowledge, the present study is the first to demonstrate that miR-22 may be a promising therapeutic target and may have potential as part of a combination treatment alongside chemotherapeutic agents for OS.

\section{Introduction}

Osteosarcoma, a primary bone malignancy, most commonly occurs during childhood and adolescence (1-3). In $20 \%$ of patients, pulmonary metastasis is clinically detected, which is the major cause of OS-associated mortality (4). At present, the standard treatment strategy for patients with OS usually consists of surgery resection and multi-drug chemotherapy, which has improved the cure rates of patients with OS by $\geq 50 \%$ since the 1970 s $(5,6)$. However, chemotherapy is associated with severe side effects and there are currently no efficient biomarkers that allow the dosage of chemotherapeutic drugs to be adapted to restrict dose-associated toxicity. Therefore, the identification of novel and effective therapeutic approaches to further improve the treatment for patients with OS is required.

MicroRNAs (miRNAs/miRs), endogenous noncoding RNAs, are 18-24 nucleotides in length and are involved in the regulation of the expression of two-thirds of all human genes through binding to mRNA 3'-untranslated regions (3'-UTRs) (7-9). miRNAs are not only implicated in the maintenance of normal cellular processes, but also act as tumor suppressors or promotors by targeting specific genes. For example, it was demonstrated that miR-21 knockdown inhibited the cell proliferation of OS cell lines, while elevated miR-21 expression accelerated the proliferation of OS cells and increased their resistance to cisplatin (10). miR-22 has been reported to be downregulated in several tumor types, including lung, liver and colorectal tumors, as well as in OS (11-13). Xia et al (11) demonstrated that miR-22 significantly attenuated OS, prostate cancer, cervical cancer and lung cancer cell proliferation and invasion. However, the mechanism by which miR-22 exerts these antitumor effects and the association with chemotherapy regimens in OS treatment remains unclear.

S100 calcium-binding protein A11 (S100A11), which is also termed calgizzarin or S100C, belongs to the S100 protein family, which are $10-12 \mathrm{kDa}$ in molecular weight and able to bind calcium by EF-hand motifs (14). S100A11 is expressed 
ubiquitously in tissues and exhibits various cellular functions, including cancer progression $(15,16)$. Previous studies have demonstrated that increased S100A11 expression is associated with tumor metastasis and a poor prognosis in pancreatic, lung and colon cancers (17-19).

The current study aimed to investigate the role of miR-22 in the carcinogenesis and progression of OS, and to determine whether modulation of miR-22 expression may affect the susceptibility of OS cells to the standard chemotherapy regimens in OS treatment.

\section{Materials and methods}

Patients and specimens. A total of 4 male and 3 female patients with OS (aged 12-22 years), and a control group consisting of 7 healthy volunteers (4 male and 3 female, aged 11-22 years), were recruited from February 2016 to February 2017 at the Second Affiliated Hospital of Xi'an Jiaotong University (Xi'an, China). Prior to the present study, none of the 7 patients with OS had received surgery, preoperative chemotherapy or radiotherapy. Patient information is presented in Table I. The ages of patients and healthy volunteers were not significantly different. OS cases were definite diagnoses based on accepted clinicopathological and radiological criteria. The study was authorized by the Ethics Committee of The Second Affiliated Hospital of Xi'an Jiaotong University (Xi'an, China). All patients and volunteers were anonymous and gave written informed consent. Blood samples were serially collected from the venous blood of patients and volunteers, which was centrifuged $\left(4^{\circ} \mathrm{C}, 600 \mathrm{x} \mathrm{g}\right.$, for $\left.10 \mathrm{~min}\right)$ and plasma was shipped on dry ice to a central repository and stored at $-80^{\circ} \mathrm{C}$ until further biochemical analysis.

Cell culture. The MG-63 human osteosarcoma and hFOB 1.19 normal osteoblast cell lines were purchased from the Cell Bank of Type Culture Collection of the Chinese Academy of Sciences (Shanghai, China). Cells were cultured at $37^{\circ} \mathrm{C}$ in $5 \% \mathrm{CO}_{2}$ in Dulbecco's modified Eagle's medium (DMEM; Gibco; Thermo Fisher Scientific, Inc., Waltham, MA, USA) supplemented with $10 \%$ fetal bovine serum (FBS; Bioind, Kibbutz Beit-Haemek, Israel), $100 \mathrm{U} / \mathrm{ml}$ penicillin/streptomycin mixed solution and $2 \mathrm{mM}$ glutamine (Beyotime Institute of Biotechnology, Haimen, China).

Cell transfection. Hsa-miR-22 overexpression plasmid (pGCMV/EGFP) and the negative control (NC, empty pGCMV/EGFP plasmid) were supplied by Shanghai GenePharma Co., Ltd. (Shanghai, China). The sequence of the miR-22 used within the plasmid is 5'-ACGGUACCC CGGCUGGGUGUU-3, the scrambled sequence was used within the NC group is 5'-ACGGUACCCCGGCUAGGG UGUC-3. The human S100A11 gene was constructed into a pcDNA3.1+HA empty vector (Invitrogen; Thermo Fisher Scientific, Inc.) and the NC of the pcDNA3.1+HA-S100A11 group was the pcDNA3.1+HA-empty group. Untransfected MG-63 cells were employed as a blank control group. For transfection, MG-63 cells were seeded in a 24-well plate at a density of $1 \times 10^{5} / \mathrm{ml}$ for $24 \mathrm{~h}$ and were subsequently transfected with $100 \mathrm{nM}$ plasmids using Lipofectamine ${ }^{\circledR} 2000$ (DNA/Lipofectamine ${ }^{\circledR} 2000=1 / 2.5$; Invitrogen; Thermo
Table I. Clinical information for patients with OS included in the current study.

\begin{tabular}{lcl}
\hline Sex & Age, years & Pathological type \\
\hline Female & 12 & Parosteal OS \\
Male & 16 & Conventional OS \\
Male & 15 & Chondroblastic OS \\
Female & 20 & Conventional OS \\
Female & 17 & Conventional OS \\
Male & 22 & Osteoblastoma OS \\
Male & 18 & Telangiectatic OS \\
\hline
\end{tabular}

OS, osteosarcoma.

Fisher Scientific, Inc.) and incubated for $6 \mathrm{~h}$ at $37^{\circ} \mathrm{C}$. After $6 \mathrm{~h}$ post-transfection, the medium was changed to $2 \%$ FBS-DMEM with blasticidin $(12 \mu \mathrm{g} / \mathrm{ml})$ for 15 days at $37^{\circ} \mathrm{C}$. Cells where stable transfection was verified were stored in liquid nitrogen for further experiments. Reverse transcription-quantitative polymerase chain reaction (RT-qPCR) and western blotting were used to determine stable transfection as described below.

Cell Counting Kit (CCK)-8 assay. The CCK-8 was supplied by Sigma-Aldrich (Merck KGaA, Darmstadt, Germany). To analyze cell proliferation, MG-63 stable transfection cells (miR-22 and miR-22+ pcDNA3.1+HA-S100A11) were seeded at a density of $8 \times 10^{3}$ cells/well in 96-well plates and incubated for $12,24,48$ or $72 \mathrm{~h}$ at $37^{\circ} \mathrm{C}$. The optical density (OD) value was measured at $480 \mathrm{~nm}$ using a Bio-Rad 2550 EIA Reader (Bio-Rad Laboratories, Inc., Hercules, CA, USA).

To analyze cell viability, MG-63 stable transfection cells (miR-22 and miR-22+ pcDNA3.1+HA-S100A11) were seeded at a density of $1 \times 10^{5}$ cells/well in 6-well plates and incubated for $24 \mathrm{~h}$ at $37^{\circ} \mathrm{C}$. Subsequently, $0,0.1,0.2$ and $0.3 \mu \mathrm{M}$ cisplatin (20) (Sigma-Aldrich; Merck KGaA) were added to the cells. After $48 \mathrm{~h}$ incubation at $37^{\circ} \mathrm{C}$ with the CCK-8 reagent, the $\mathrm{OD}$ values were measured and relative cell viability was obtained by setting corresponding untreated groups as $100 \%$. Half maximal inhibitory concentration $\left(\mathrm{IC}_{50}\right)$ values were calculated using GraphPad Prism 6.0 software (GraphPad Software, Inc., La Jolla, CA, USA).

Transwell migration assay. A Transwell migration assay was used to determine cell migration ability. A total of $\sim 10^{3}$ MG-63 stable transfection cells (miR-22 and miR-22 + pcDNA3.1+HA-S100A11) were cultured with DMEM medium in the upper chamber of 24 -well plates, and the lower chamber was filled with $20 \%$ FBS-DMEM medium as a chemoattractant. After incubation for $48 \mathrm{~h}$ at $37^{\circ} \mathrm{C}$, cells that migrated to the lower side of the inserts were fixed with $4 \%$ paraformaldehyde at room temperature for 20 min and stained with $0.1 \%$ crystal violet (Beyotime Institute of Biotechnology) at room temperature for $20 \mathrm{~min}$, and then the cells from five independent, randomly chosen visual fields were counted under a light microscope (magnification, x100; Nikon Corporation, Japan) for quantification of cells. 
A

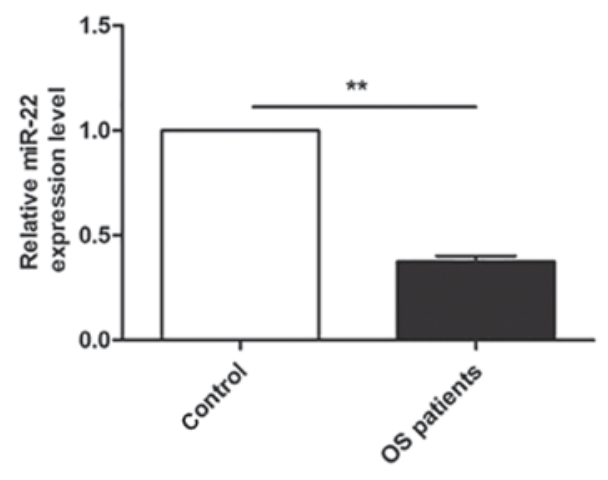

B

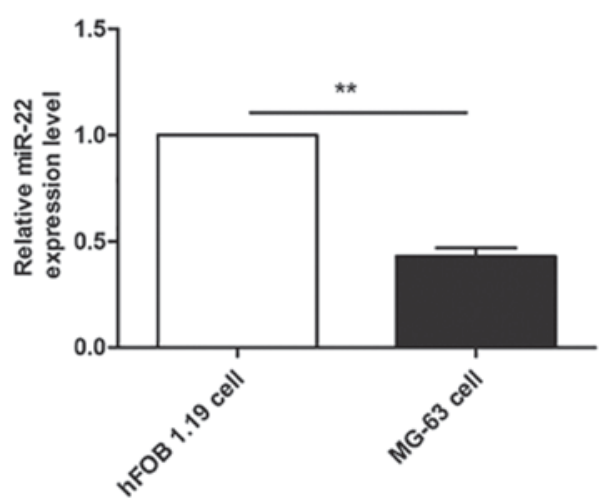

Figure 1. Endogenous expression of miR-22 in patients with OS and an OS cell line. (A) Serum expression of miR-22 was validated to be lower in patients with OS compared with the healthy volunteer group. (B) miR-22 levels were significantly lower in MG-63 OS cells compared with hFOB 1.19 normal osteoblast cells. ${ }^{* *} \mathrm{P}<0.01$, as indicated. miR, microRNA; OS, osteosarcoma.

$R T-q P C R$. Isolation of the total RNA from serum or cells was performed using an RNeasy mini-kit (Qiagen $\mathrm{GmbH}$, Hilden, Germany) and single-strand cDNA was reverse-transcribed by using ThermoScript One Step RT-PCR kit (Thermo Fisher Scientific, Inc.) following the manufacturer's instructions (Invitrogen; Thermo Fisher Scientific, Inc.). qPCR was performed on a real-time quantified PCR system with a Rotor-Gene SYBR-Green PCR kit (400) (Qiagen China Co., Ltd., Shanghai, China) on an ABI PRISM 7500 Real-Time PCR System (Applied Biosystems; Thermo Fisher Scientific, Inc.) with the following steps: Step 1: Predenaturation at $95^{\circ} \mathrm{C}$ for $5 \mathrm{~min}$; step 2: 40 cycles of denaturation at $95^{\circ} \mathrm{C}$ for $10 \mathrm{sec}$, then annealing and extension at $60^{\circ} \mathrm{C}$ for $34 \mathrm{sec}$. The experiments were repeated three times. The relative miRNA or mRNA expression levels were determined using the $2^{-\Delta \Delta \mathrm{Cq}}$ method (21) and normalized to U6 and GAPDH, respectively. The reverse transcription primer for miR-22 was 5'-CCAGCTAAAGCT GCCAGTTGAAGAACTG-3'. The qPCR primers were as follows: miR-22, 5'-AAGCUGCCAGUUGAAGAACUGU-3' (forward) and 5'-AGUUCUUCAACUGGCAGCUUUU-3' (reverse); U6, 5'-CTCGCTTCGGCAGCACA-3' (forward) and 5'-AACGCTTCACGAATTTGCGT-3' (reverse); S100A11, 5'-TCTCCAAGACAGAGTTCCTAAGC-3' (forward) and 5'-TCATGCGGTCAAGGACAC-3' (reverse); and GAPDH, 5'-GAAACCAGATCTCCACCGCA-3' (forward) and 5'-GCG CCCAATACGACCAAATC-3' (reverse).

Western blot analysis. Cells were harvested and extracted using radioimmunoprecipitation assay lysis buffer (Beyotime Institute of Biotechnology). Protein concentration was determined via a Bradford's assay. Denatured cell lysates $(30 \mu \mathrm{g})$ were resolved by $8 \%$ SDS-PAGE, transferred to nitrocellulose membranes. The membrane was blocked with the blocking buffer for $30 \mathrm{~min}$ at room temperature, and then incubated overnight with antibodies against marker of proliferation Ki-67 (1:1,000; ab15580, Abcam, Cambridge, UK), proliferating cell nuclear antigen (PCNA; 1:1,000; ab29; Abcam), matrix metallopeptidase MMP2 (1:1,000; ab37150; Abcam) and GAPDH (1:1,000, ab8245; Abcam). Tris-buffered saline with $0.05 \%$ Tween-20 was used to wash the membranes three times for $10 \mathrm{~min}$. Subsequently, the membranes were incubated with a secondary antibody, rabbit horseradish peroxidase-conjugated anti-goat immunoglobulin G (1:2,000; sc2771; Santa Cruz Biotechnology Inc., Dallas, TX, USA) for $1 \mathrm{~h}$ at room temperature. A chemiluminescence system (ECL Plus; Odyssey Infrared Imaging system; LI-COR Biosciences, Lincoln, NE, USA) was used for visualization. The bands were scanned on a gel imaging and analysis system and analyzed by Quantity One 4.4 (Bio-Rad Laboratories, Inc.).

ELISA. A Quantikine ELISA kit (KA2413; R\&D Systems, Inc., Minneapolis, MN, USA) was used to detect the expression of S100A11 protein. Twenty-four hours post-transfection, the cell supernatants of different MG-63 cell treatment groups or serum from patients were collected and analyzed according to the manufacturer's protocol.

Reporter gene transfection and luciferase assays. TargetScan (http://www.targetscan.org/vert_71/) and miRanda (http://34.236.212.39/microrna/home.do) databases were used to predict the potential miR-22 binding sites of S100A11. In the luciferase reporter assay, $1 \times 10^{6} \mathrm{MG}-63$ cells/well that were stably transfected with miR-22 were further transfected with $30 \mathrm{ng}$ wild-type or mutant 3'-UTR of S100A11 via Lipofectamine ${ }^{\circledR} 2000$ (Invitrogen; Thermo Fisher Scientific, Inc.). After $48 \mathrm{~h}$, cells were collected and luciferase activity was measured by a Dual-Luciferase Reporter Assay System (Promega Corporation, Madison, WI, USA) according to the manufacturer's protocol.

The S100A11 3'-UTR was cloned into a luciferase pMIR-REPORT vector (Shanghai GeneChem Co., Ltd., Shanghai, China) and mutations were introduced in potential miR-22 binding sites. Values were double normalized to firefly luciferase activity. PRL-SV40 vector carrying the Renilla luciferase gene (Promega Corporation) was used as an internal control for transfection efficiency. The sequences included: S100A11 3'-UTR, 5'-UCUGAGUUCUUGAAGCAUUUC AA-3', hsa-miR-22, 3'-GAUCACCAGGAUUUGUAAAGU G-5' and S100A11 3'-UTR, 5'-UCUGAGUUCUUGAAGAUC GAUCA-3'.

Statistical analysis. Experiments were performed at least three independent times and data are presented as the mean \pm standard deviation. Statistical significance was 

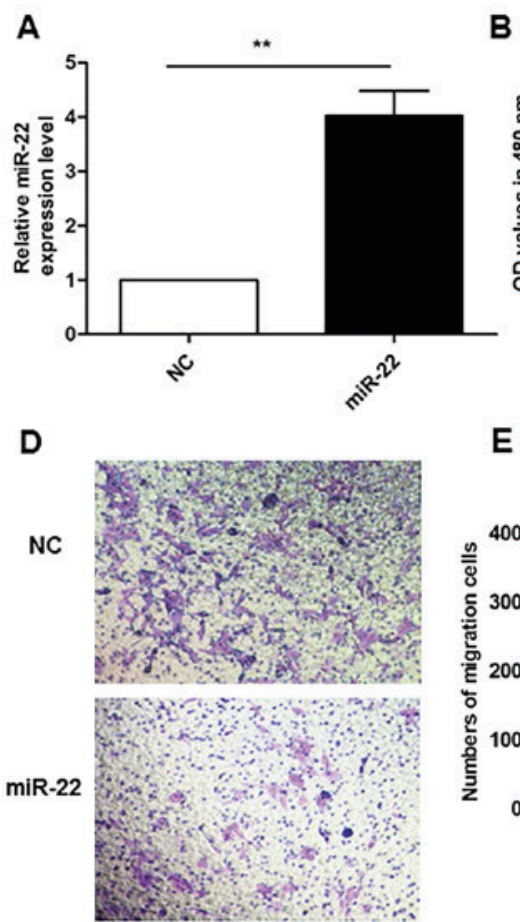

(x100 magnification)
B

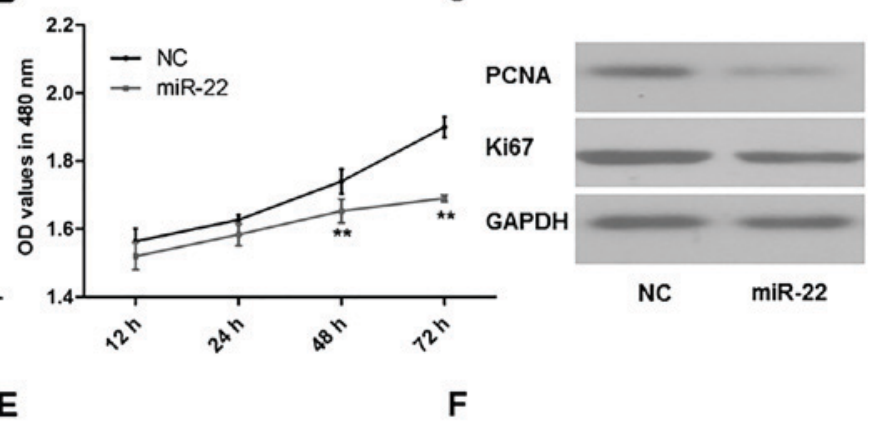

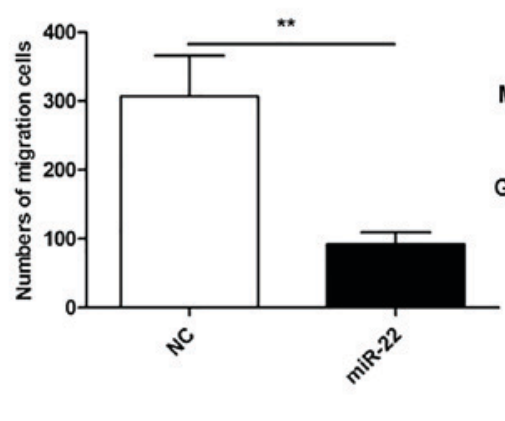

MMP2

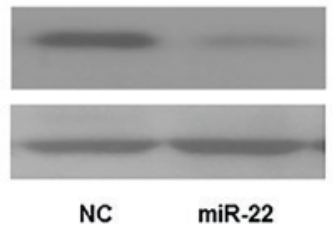

Figure 2. miR-22 overexpression inhibits the proliferation and migration ability of MG-63 cells. (A) miR-22 expression was significantly increased in MG-63 cells following transfection with miR-22. (B) miR-22 markedly inhibited the proliferation ability of MG- 63 cells at 48 and $72 \mathrm{~h}$. (C) miR-22 reduced the protein expression levels of Ki67 and PCNA. (D) Representative images of lower chambers following Transwell migration assays with MG-63 cells. Magnification, x100. (E) Migration assay results were quantified and miR-22 significantly inhibited the migratory ability of MG-63 cells. (F) miR-22 reduced the protein expression levels of MMP2 compared with the NC group. ${ }^{* *} \mathrm{P}<0.01$. miR, microRNA; Ki67, marker of proliferation Ki-67; PCNA, proliferating cell nuclear antigen; MMP2, matrix metallopeptidase 2 ; NC, negative control; OD, optical density.

evaluated by one-way analysis of variance followed by a Tukey's post hoc test or two-tailed Student's t-tests using SPSS 20.0 software (IBM Corp., Armonk, NY, USA) and GraphPad Prism 6.0 software. $\mathrm{P}<0.05$ was considered to indicate a statistically significant difference.

\section{Results}

Endogenous expression of $m i R-22$ in patients with $O S$ and an OS cell line. The expression of miR-22 from the serum of patients with OS and healthy controls, and in vitro cultured OS and normal osteoblast cells, was investigated by RT-qPCR. As demonstrated in Fig. 1A, the serum expression of miR-22 was lower in samples from patients with OS compared with healthy volunteers $(\mathrm{P}<0.05)$. Furthermore, Fig. 1B also indicated that the miR-22 levels were $\sim$ two fold lower in the MG-63 OS cell line compared with the hFOB 1.19 osteoblast cell line $(\mathrm{P}<0.01)$.

miR-22 overexpression inhibits the proliferation and migration of MG-63 Cells. As demonstrated in Fig. 2A, MG-63 cells were successfully transfected with miR-22 overexpression plasmids, and qPCR results indicated that the levels of miR-22 were $\sim 4$-fold higher than those in the NC group $(\mathrm{P}<0.01)$. A CCK-8 assay was performed to investigate cell proliferation. The results demonstrated that overexpression of miR-22 markedly inhibited the proliferation of MG-63 cells at 48 and 72 h, compared with the NC group ( $\mathrm{P}<0.01$; Fig. 2B). In addition, miR-22 overexpression also reduced the protein expression levels of Ki67 and PCNA compared with the NC group (Fig. 2C).

A Transwell migration assay was performed to determine the effects of miR-22 on cell migration ability. The results demonstrated that overexpression of miR-22 resulted in the inhibition of MG-63 cell migration, compared with the $\mathrm{NC}$ group $(\mathrm{P}<0.01$; Fig. $2 \mathrm{D}$ and $\mathrm{E})$. In accordance with migration results, miR-22 overexpression also reduced the protein expression levels of MMP2 compared with the NC group (Fig. 2F).

miR-22 overexpression sensitizes MG-63 cells to cisplatin treatment and reduces the expression of S100A11. As cisplatin is frequently used clinically for OS treatment (22), the current study assessed whether modulated miR-22 levels in MG-63 cells may affect the sensitivity of cells to cisplatin chemotherapy. The results in Fig. 3A and B demonstrate that the cell viability of MG-63 cells that overexpress miR-22 was decreased in a dose-dependent manner for cisplatin treatment; overexpression of miR-22 increased the sensitivity of MG-63 to cisplatin and resulted in a significant reduction in the calculated $\mathrm{IC}_{50}$ value, compared with the $\mathrm{NC}$ group $(\mathrm{P}<0.01)$. The mRNA expression of S100A11 in transfected cells, and the levels of S100A11 in the cell culture medium, was also investigated. The results in Fig. 3C and D demonstrated that reduced mRNA levels of S100A11, and reduced release of S100A11 protein into the culture medium, were observed in MG-63 cells with miR-22 overexpression, compared with the NC group $(\mathrm{P}<0.01)$. 
A
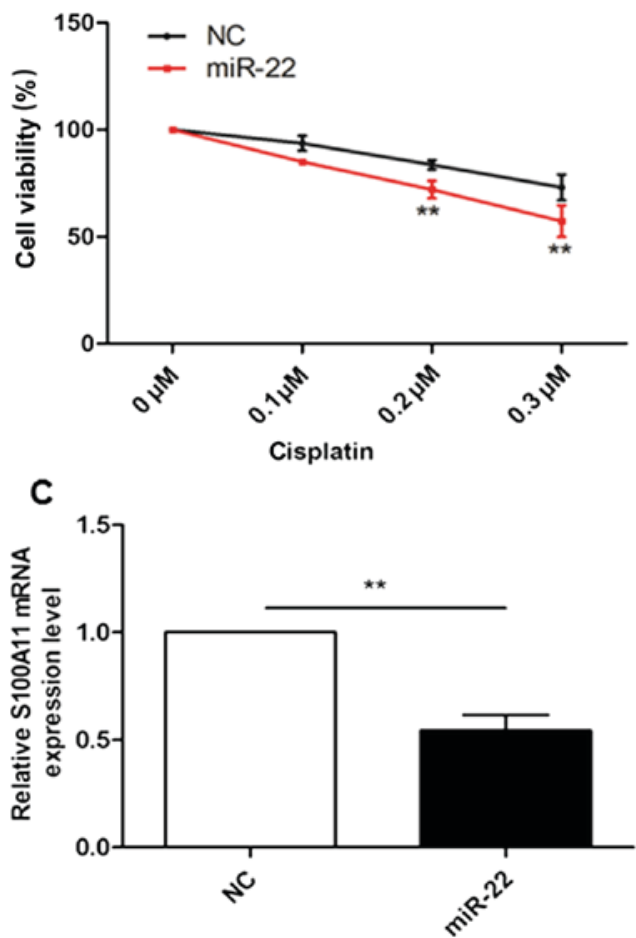

B
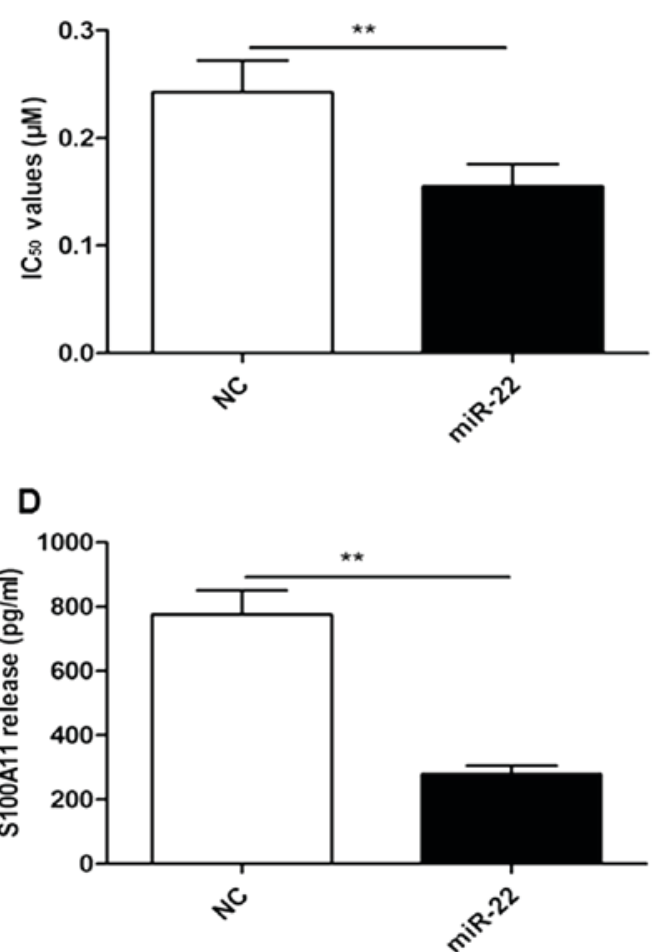

Figure 3. miR-22 overexpression sensitizes MG-63 cells to cisplatin treatment and decreases the levels of S100A11. (A) Cell viability of MG-63 cells transfected with miR-22 was decreased in a dose-dependent manner when exposed to increasing concentrations of cisplatin $(0,0.1,0.2$ and $0.3 \mu \mathrm{M})$. (B) Overexpression of miR-22 significantly reduced the calculated IC50 values in MG-63 cells. (C) S100A11 mRNA levels in MG-63 cells and (D) S100A11 protein release by MG-63 cells into the cell culture medium were decreased significantly following transfection with miR-22. For part A, ${ }^{* * *} \mathrm{P}<0.01$ vs. NC group at same time-point; for parts B-D, ${ }^{* *} \mathrm{P}<0.01$, as indicated. miR, microRNA; S100A11, S100 calcium-binding protein A11; $\mathrm{IC}_{50}$, half maximal inhibitory concentration; NC, negative control.

A

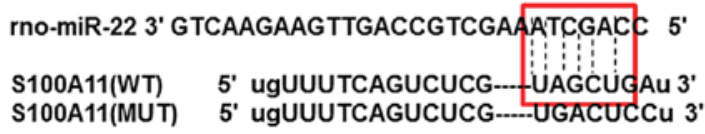

B

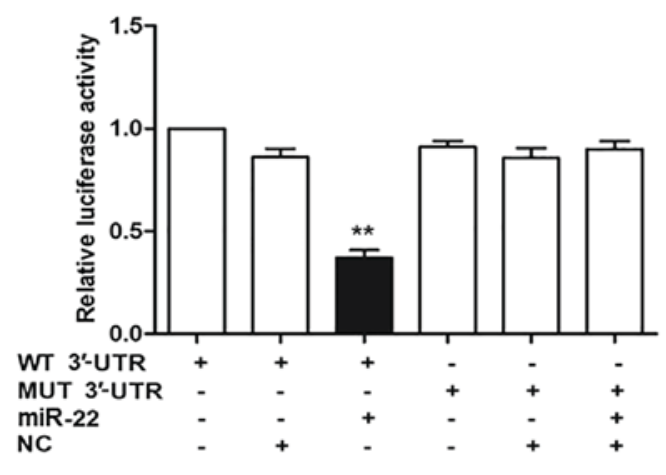

C

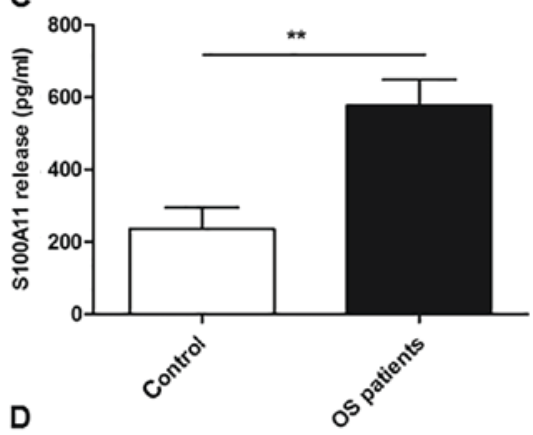

D

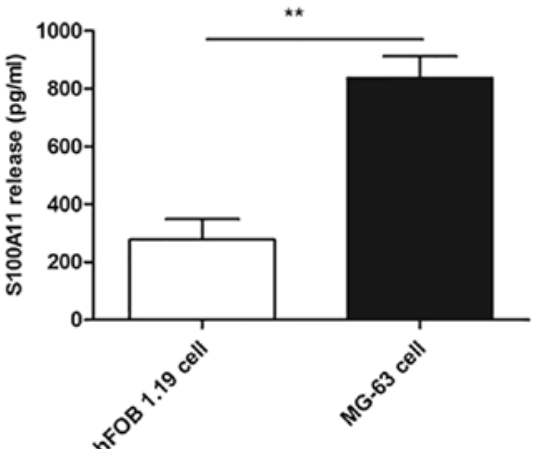

Figure 4. S100A11 is a direct target of miR-22. (A) Sequences of WT S100A11 3'-UTR and MUT S100A11 3'-UTR, and the ability of miR-22 to bind to the WT sequence. (B) Relative luciferase activity in MG-63 cells following co-transfection of miR-22 or NC and the WT or MUT S100A11 3'-UTR. Levels of S100A11 in (C) the serum of patients with OS and healthy volunteers, and (D) the cell culture medium of MG-63 OS cells and hFOB 1.19 normal osteoblasts, were determined by ELISA. For part B, ${ }^{* *} \mathrm{P}<0.01$ vs. MG-63 cells co-transfected with NC and the WT $3^{\prime} \mathrm{UTR}$ of S100A11; for parts C and D, ${ }^{* *} \mathrm{P}<0.01$, as indicated. S100A11, S100 calcium-binding protein A11; miR, microRNA; WT, wild-type; UTR, untranslated region; MUT, mutant; NC, negative control; OS, osteosarcoma. 
A

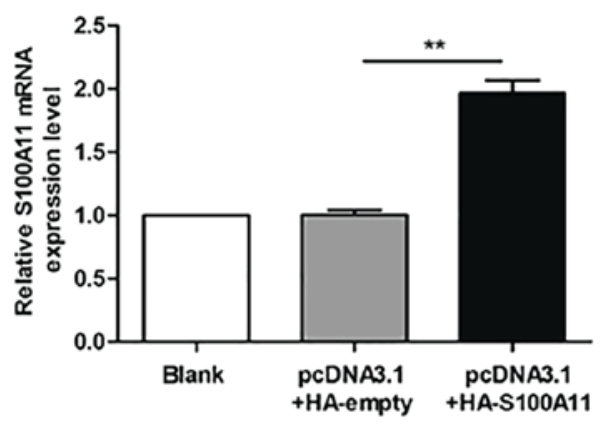

C

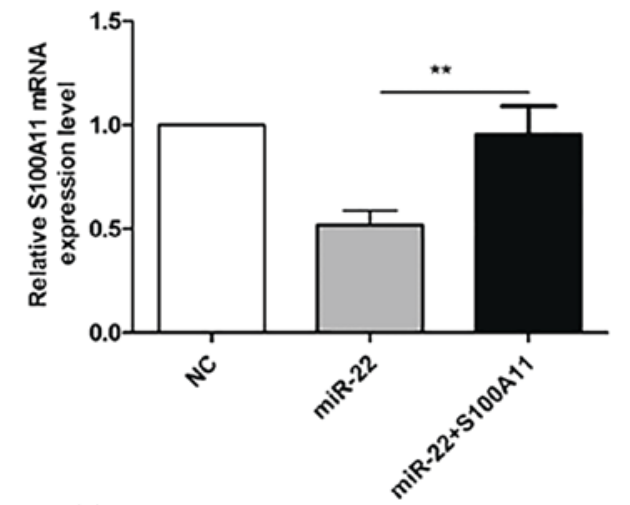

$\mathbf{E}$

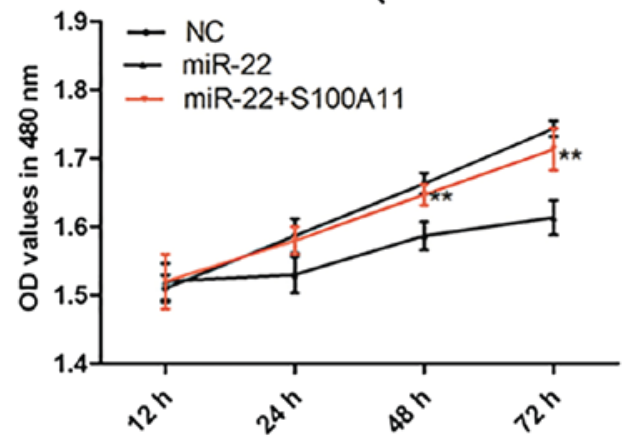

B

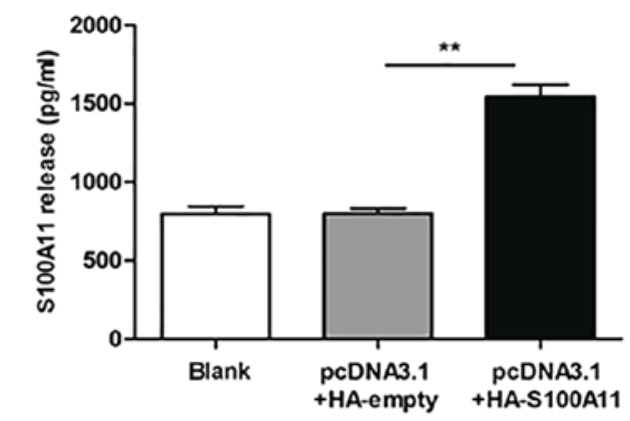

D

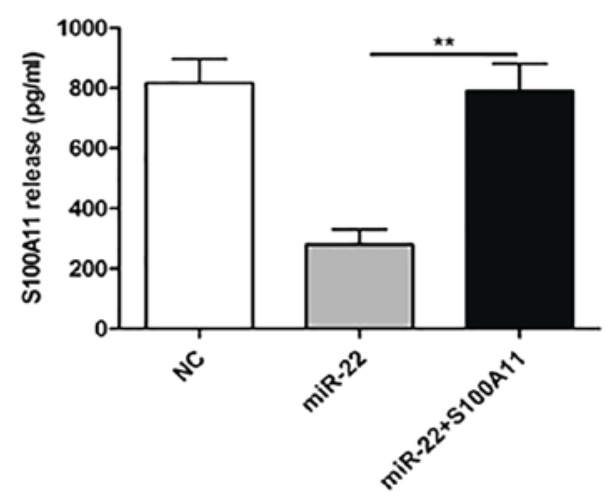

$\mathbf{F}$

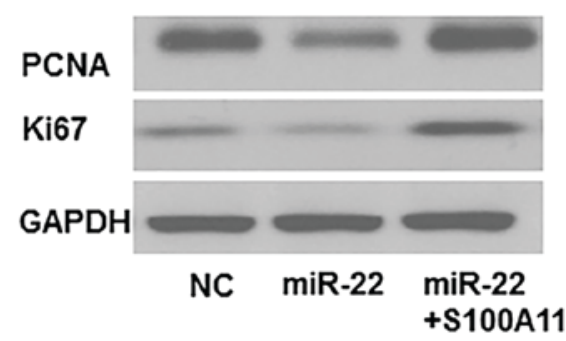

Figure 5. S100A11 is successfully overexpressed in MG-63 cells following transfection. (A) S100A11 mRNA levels in MG-63 cells and (B) S100A11 protein release by MG-63 cells into the cell culture medium were significantly increased in MG-63 cells transfected with pcDNA3.1 + HA-S100A11 compared with the pcDNA3.1 + HA-empty group. (C) S100A11 mRNA levels in MG-63 cells and (D) S100A11 protein release by MG-63 cells into the cell culture medium were increased in cells co-transfected with miR-22 and pcDNA3.1 + HA-S100A11 compared with those only transfected with miR-22. (E) Overexpression of S100A11 reversed the anti-proliferative effect of miR-22. (F) Ki67 and PCNA protein levels were increased in MG-63 cells that were co-transfected with miR-22 and pcDNA3.1 + HA-S100A11 compared with those only transfected with miR-22. For parts A-D, ${ }^{* * *} \mathrm{P}<0.01$, as indicated; for part E ${ }^{* *} \mathrm{P}<0.01 \mathrm{vs}$. miR-22 + S100A11 and NC groups at same time-point. S100A11, S100 calcium-binding protein A11; miR, microRNA; Ki67, marker of proliferation Ki-67; PCNA, proliferating cell nuclear antigen; NC, negative control; OD, optical density.

S100A11 is a direct target of miR-22. The present study employed bioinformatics tools to predict the putative miRNAs targeting S100A11, and identified that miR-22 possesses the conserved binding sites (Fig. 4A). Subsequently, a luciferase reporter gene assay was performed to verify whether miR-22 directly binds to the 3'-UTR of S100A11 in OS. The results in Fig. 4B indicate that co-transfection of wild-type S100A11 3'-UTR with miR-22 overexpression reduced the relative luciferase activity by $\sim 50 \%$, compared with NC (miR-NC)+wild-type 3'-UTR of S100A11 group. Furthermore, co-transfection of cells with the mutant 3 '-UTR of S100A11 and miR-22 overexpression reversed this suppressive effect of miR-22 $(\mathrm{P}<0.01)$. Additionally, the results in Fig. 4C and D indicate that the release of S100A11 into the serum or cell culture medium of patients with OS or MG-63 cells was significantly increased compared with healthy volunteers or hFOB 1.19 normal osteoblast cells, respectively $(\mathrm{P}<0.01)$.
Successful overexpression of S100A11 in MG-63 cells. As demonstrated in Fig. 5A and B, MG-63 cells were successfully transfected with pcDNA3.1+HA-S100A11, and RT-qPCR and ELISA results demonstrated that the levels of S100A11 were $\sim 2$-fold of those in the pcDNA3.1+HA-empty group $(\mathrm{P}<0.01)$.

S100A11 reverses the reduction in proliferation induced by overexpression of miR-22 in MG-63 cells. To investigate the functional mediator role of S100A11 for miR-22 in OS cells, the present study overexpressed S100A11 in MG-63 cells in combination with miR-22 overexpression. The results in Fig. 5C and D demonstrate that the levels of S100A11 mRNA in MG-63 cells, and S100A11 levels in the cell culture medium, were increased in the miR-22 overexpression+S100A11 overexpression group compared with cells where only miR-22 was overexpressed $(\mathrm{P}<0.01)$. As indicated in Fig. 5E, miR-22 overexpression led to reduced MG-63 cell proliferation 
A

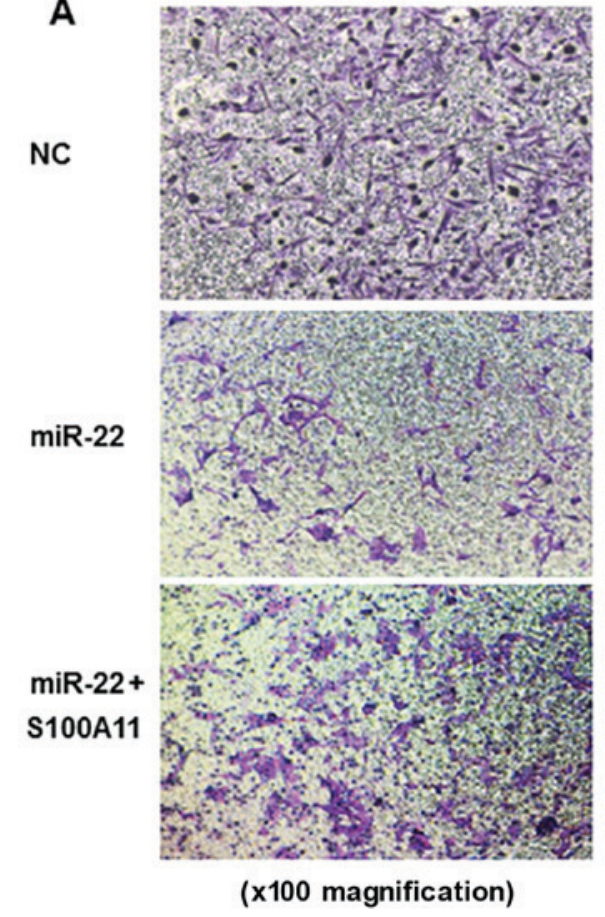

B

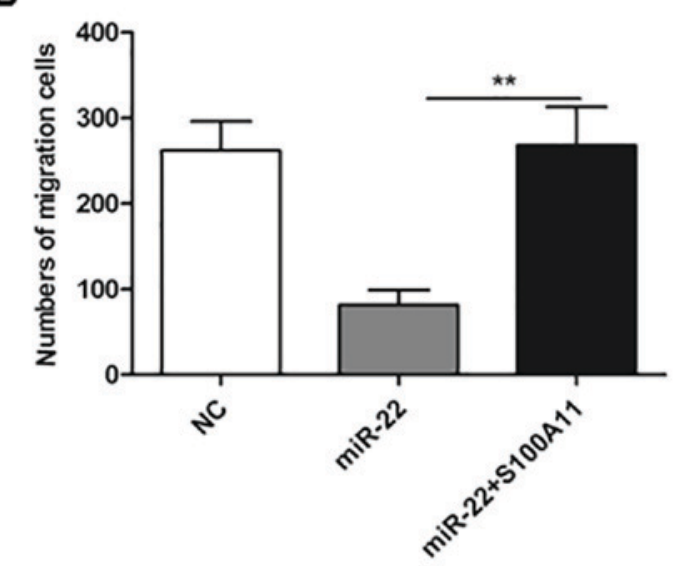

C

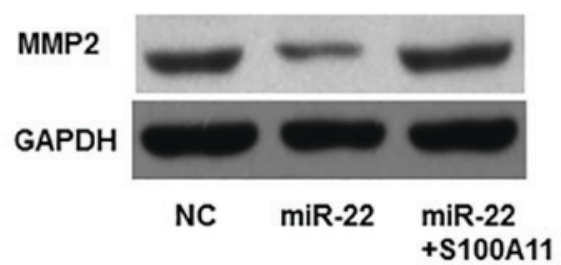

Figure 6. S100A11 overexpression reverses the reduction in migratory ability induced by overexpression of miR-22 in MG-63 cells. (A) Representative images of lower chambers following Transwell migration assays with transfected MG-63 cells. Magnification, x100. (B) Quantified migration assay results demonstrated that S100A11 overexpression reversed the inhibitory effect of miR-22 on MG-63 cell migration. (C) Protein expression of MMP2 was increased in MG-63 cells that were co-transfected with miR-22 and pcDNA3.1 + HA-S100A11 compared with those only transfected with miR-22. ${ }^{* *} \mathrm{P}<0.01$, as indicated. S100A11, S100 calcium-binding protein A11; miR, microRNA; MMP2, matrix metallopeptidase; NC, negative control.

A

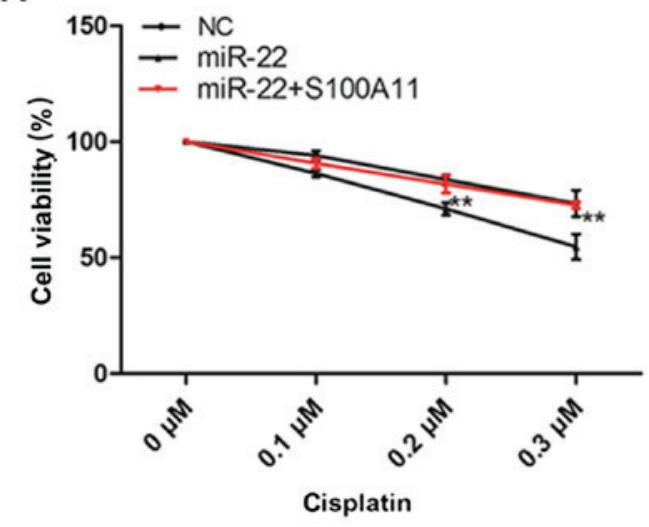

B

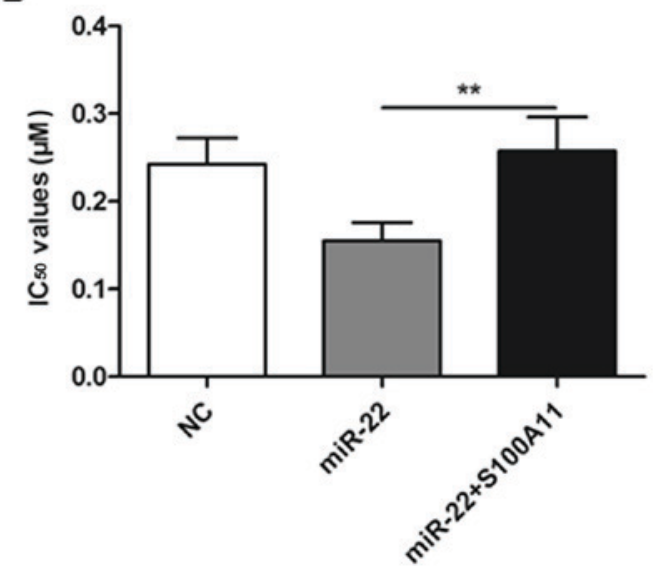

Figure 7. S100A11 desensitizes MG-63 cells to cisplatin treatment. (A) Cell viability of MG-63 cells that overexpressed miR-22 and S100A11 was increased at 0.2 ad $0.3 \mu \mathrm{M}$ cisplatin compared with those that only overexpressed miR-22. (B) S100A11 desensitized MG-63 cells to cisplatin and resulted in a significant increase in the calculated IC50 value, compared with those that only overexpressed miR-22. For part A, ${ }^{* *} \mathrm{P}<0.01$ vs. miR-22+S100A 11 and miR-22 groups at the same time-point; for part B, ${ }^{* *} \mathrm{P}<0.01$, as indicated. S100A11, S100 calcium-binding protein A11; miR, microRNA; $\mathrm{IC}_{50}$, half maximal inhibitory concentration; NC, negative control.

compared with the NC group, while simultaneous S100A11 overexpression reversed the anti-proliferative effect of miR-22 $(\mathrm{P}<0.01)$. In addition, miR-22-induced reductions in the protein expression of PCNA and Ki67 were reversed in co-transfected cells (Fig. 5F).

S100A11 reverses miR-22-induced reductions in the migratory ability of MG-63 cells. Results in Fig. 6A and B demonstrate that S100A11 overexpression reversed the inhibitory effect of miR-22 on MG-63 cell migration $(\mathrm{P}<0.01)$. In addition, the expression of MMP2 in co-transfected cells was increased compared with cells that only overexpressed miR-22 (Fig. 6C).

S100A11 desensitizes MG-63 cells to cisplatin treatment. As demonstrated in Fig. 7, the cell viability of MG-63 cells that were co-transfected with miR-22 and S100A11 decreased in 
a dose-dependent manner for cisplatin treatment; S100A11 desensitized MG-63 cells to cisplatin and resulted in a significant increase in the calculated $\mathrm{IC}_{50}$ value, compared with cells that only overexpressed miR-22 $(\mathrm{P}<0.01)$.

\section{Discussion}

The present study observed that the expression levels of miR-22 were significantly reduced in patients with OS and the MG-63 OS cell line compared with healthy volunteers and the hFOB 1.19 normal osteoblast cell line, respectively. In addition, the expression of miR-22 was inversely associated with S100A11 levels, which was significantly upregulated in patients with OS and MG-63 cells, compared with the respective controls. In vitro results demonstrated that overexpression of miR-22 inhibited the proliferation and migratory ability, and increased the sensitivity to cisplatin treatment, in MG-63 cells. Notably, the present study identified S100A11 as a direct target gene of miR-22 in MG-63 cells. Further experiments demonstrated that the alterations in proliferation, migration and chemosensitivity were partially abolished by overexpression of the S100A11 gene. Therefore, the current study revealed that S100A11 is a specific target gene for miR-22 and that this miRNA may function as a tumor suppressor in OS through enhancing the chemosensitivity of cells to cisplatin.

OS often occurs in children and adolescents (23); the 5 -year survival rate for OS remains $<70 \%$ for OS cases that are most resistant to therapies (24). miRNAs have been implicated in the development of chemosensitivity and chemoresistance, which may aid the understanding of the molecular basis of this aggressive bone cancer $(25,26)$. Recently, scientists have reported that several miRNAs may be involved in the sensitivity of cancers to chemotherapeutic agents. Vanas et al (10) demonstrated that reduced miR-21 expression inhibited the proliferation of OS cells lines, while elevated miR-21 expression increased the proliferation and overexpression of miR-21 increased the resistance of cells to cisplatin treatment. Furthermore, Geng et al (20) reported an inverse association between miR-224 and Rac family small GTPase 1 (Rac1); miR-224 inhibited the proliferation of OS cell lines, as well as their metastasis and sensitivity to cisplatin treatment, through direct targeting of Racl. In addition, miR-138 was also reported to be involved in cisplatin resistance. Research demonstrated that miR-138 was reduced in OS tissue and restoring miR-138 expression markedly inhibited cell proliferation and invasion, and increased sensitivity to cisplatin (27). These findings confirmed that miRNAs affect the sensitivity of cancers to chemotherapeutic agents. However, to the best of our knowledge, no previous studies have described the effect of miR-22 in the modulation of sensitivity to cisplatin in OS. The present study, the present study demonstrated for the first time that miR-22 may be a promising therapeutic target and may have potential for use in combination with chemotherapeutic agents.

S100A11, a member of the S100 protein family, is widely expressed in human tissues. Increased levels of S100A11 have been reported in various cancer types and was closely associated with tumor progression $(28,29)$. S100A11 has been proposed to exhibit pleiotropic effects in various biological contexts, including in nuclear and cytoplasmic compartments, and S100A11 has been reported to act as a growth inhibitor in normal keratinocytes $(30,31)$. Furthermore, when the protein was secreted in dimerized form and bound to receptor for advanced glycation end products, S100A11 induced the growth stimulation in normal keratinocytes and various cancer cells (32). At present, S100A11 in OS has not been fully characterized, and the present study aimed to investigate the role of this protein in OS progression. The results of the current study indicated that S100A11 was highly upregulated in the serum of patients with OS and the culture medium of the MG-63 OS cell line compared with respective controls, and further research identified S100A11 as a specific target gene of miR-22. These novel findings may aid the understanding of the extracellular role of dimerized S100A11 in the OS cell microenvironment and its association with cancer progression.

In conclusion, the present study demonstrated that levels of miR-22 were significantly reduced in patients with and the MG-63 OS cell line, while the expression of S100A11 was inversely associated with miR-22 levels. Overexpression of miR-22 inhibited the proliferation and migration, and increased the sensitivity to cisplatin treatment, in MG-63 cells. However, overexpression of S100A11 partially abolished the alterations in proliferation, migration and chemosensitivity that were induced by miR-22. Furthermore, the present study identified S100A11 as a direct target gene of miR-22 in MG-63 cells. The current study demonstrated for the first time that miR-22 may be a promising therapeutic target and may have potential in combination with chemotherapeutic agents for OS.

\section{Acknowledgements}

Not applicable.

\section{Funding}

No funding was received.

\section{Availability of data and materials}

The datasets used and/or analyzed during the current study are available from the corresponding author on reasonable request.

\section{Authors' contributions}

$\mathrm{XH}$ designed the experiment, XZho conducted the experimental and statistical work and wrote the paper. DN, XZha and ZG helped XZho conduct the cell experiments.

\section{Ethics approval and consent to participate}

The present study was authorized by the Ethics Committee of The Second Affiliated Hospital of Xi'an Jiaotong University (Xi'an, China). All patients and volunteers were anonymous and provided written informed consent.

\section{Consent for publication}

Not applicable. 


\section{Competing interests}

The authors declare that they have no competing interests.

\section{References}

1. Messerschmitt PJ, Garcia RM, Abdul-Karim FW, Greenfield EM and Getty PJ: Osteosarcoma. J Am Acad OrthopSurg 17: 515-527, 2009.

2. Bousquet M, Noirot C, Accadbled F, Sales de Gauzy J, Castex MP, Brousset $\mathrm{P}$ and Gomez-Brouchet A: Whole-exome sequencing in osteosarcoma reveals important heterogeneity of genetic alterations. Ann Oncol 27: 738-744, 2016.

3. Mirabello L, Troisi RJ and Savage SA: International osteosarcoma incidence patterns in children and adolescents, middle ages and elderly persons. Int J Cancer 125: 229-234, 2009

4. Miller BJ, Cram P, Lynch CF and Buckwalter JA: Risk factors for metastatic disease at presentation with osteosarcoma: An analysis of the SEER database. J Bone Joint Surg Am 95: e89, 2013.

5. Ferrari S and Palmerini E: Adjuvant and neoadjuvant combination chemotherapy for osteogenic sarcoma. Curr Opin Oncol 19: 341-346, 2007

6. Allison DC, Carney SC, Ahlmann ER, Hendifar A, Chawla S, Fedenko A, Angeles C and Menendez LR: A meta-analysis of osteosarcoma outcomes in the modern medical era. Sarcoma 2012: 704872, 2012.

7. Lee RC and Ambros V: An extensive class of small RNAs in Caenorhabditis elegans. Science 294: 862-864, 2001.

8. Friedman RC, Farh KK, Burge CB and Bartel DP: Most mammalian mRNAs are conserved targets of microRNAs. Genome Res 19: 92-105, 2009.

9. Irwandi RA and Vacharaksa A: The role of microRNA in periodontal tissue: A review of the literature. Arch Oral Biol 72 66-74, 2016.

10. Vanas V, Haigl B, Stockhammer V and Sutterlüty-Fall H: MicroRNA-21 increases proliferation and cisplatin sensitivity of osteosarcoma-derived cells. PLoS One 11: e0161023, 2016

11. Xia SS, Zhang GJ, Liu ZL, Tian HP, He Y, Meng CY, Li LF, Wang ZW and Zhou T: MicroRNA-22 suppresses the growth, migration and invasion of colorectal cancer cells through a Sp1 negative feedback loop. Oncotarget 8: 36266-36278, 2017.

12. Qiao DD, Yang J, Lei XF, Mi GL, Li SL, Li K, Xu CQ and Yang HL: Expression of microRNA-122 and microRNA-22 in HBV-related liver cancer and the correlation with clinical features. Eur Rev Med Pharmacol Sci 21: 742-747, 2017.

13. Chen J, Wu FX, Luo HL, Liu JJ, Luo T, Bai T, Li LQ and Fan XH: Berberine upregulates miR-22-3p to suppress hepatocellular carcinoma cell proliferation by targeting Spl. Am J Transl Res 8 : 4932-4941, 2016

14. Shankar J, Messenberg A, Chan J, Underhill TM, Foster LJ and Nabi IR: Pseudopodial actin dynamics control epithelial-mesenchymal transition in metastatic cancer cells. Cancer Res 70: 3780-3790, 2010

15. Jaiswal JK, Lauritzen SP, Scheffer L, Sakaguchi M, Bunkenborg J, Simon SM, Kallunki T, Jäättelä M and Nylandsted J: S100A11 is required for efficient plasma membrane repair and survival of invasive cancer cells. Nat Commun 5: 3795, 2014.

16. Woo T, Okudela K, Mitsui H, Tajiri M, Rino Y, Ohashi K and Masuda M: Up-Regulation of S100A11 in lung adenocarcinoma-its potential relationship with cancer progression. PLoS One 10: e0142642, 2015.

17. Xiao MB, Jiang F, Ni WK, Chen BY, Lu CH, Li XY and Ni RZ: High expression of S100A11 in pancreatic adenocarcinoma is an unfavorable prognostic marker. Med Oncol 29: 1886-1891, 2012.
18. Tian T, Hao J, Xu A, Hao J, Luo C, Liu C, Huang L, Xiao X and He D: Determination of metastasis-associated proteins in non-small cell lung cancer by comparative proteomic analysis. Cancer Sci 98: 1265-1274, 2007.

19. Melle C, Ernst G, Schimmel B, Bleul A and von Eggeling F: Colon-derived liver metastasis, colorectal carcinoma, and hepatocellular carcinoma can be discriminated by the $\mathrm{Ca}^{2+}$-binding proteins S100A6 and S100A11. PLoS One 3: e3767, 2008.

20. Geng S, Gu L, Ju F, Zhang H, Wang Y, Tang H, Bi Z and Yang C: MicroRNA-224 promotes the sensitivity of osteosarcoma cells to cisplatin by targeting Rac1. J Cell Mol Med 20: 1611-1619, 2016.

21. Livak KJ and Schmittgen TD: Analysis of relative gene expression data using real-time quantitative PCR and the $2^{-\Delta \Delta C_{\mathrm{T}}}$ method. Methods 25: 402-408, 2001.

22. Gorlick R: Current concepts on the molecular biology of osteosarcoma. Cancer Treat. Res 152: 467-478, 2009.

23. Bacci G, Briccoli A, Rocca M, Ferrari S, Donati D, Longhi A, Bertoni F, Bacchini P, Giacomini S, Forni C, et al: Neoadjuvant chemotherapy for osteosarcoma of the extremities with metastases at presentation: Recent experience at the Rizzoli Institute in 57 patients treated with cisplatin, doxorubicin, and a high dose of methotrexate and ifosfamide. Ann Oncol 14: 1126-1134, 2003.

24. Yu X, Li Z, Yu J, Chan MT and Wu WK: MicroRNAs predict and modulate responses to chemotherapy in colorectal cancer. Cell Prolif 48: 503-510, 2015.

25. Song B, Wang Y, Xi Y, Kudo K, Bruheim S, Botchkina GI, Gavin E, Wan Y, Formentini A, Kornmann M, et al: Mechanism of chemoresistance mediated by miR-140 in human osteosarcoma and colon cancer cells. Oncogene 28: 4065-4074, 2009

26. Martinez-Sanchez A, Dudek KA and Murphy CL: Regulation of human chondrocyte function through direct inhibition of cartilage master regulator SOX9 by microRNA-145 (miRNA-145). J Biol Chem 287: 916-924, 2012.

27. Zhu Z, Tang J, Wang J, Duan G, Zhou L and Zhou X: MiR-138 acts as a tumor suppressor by targeting EZH2 and enhances cisplatin-induced apoptosis in osteosarcoma cells. PLoS One 11: e0150026, 2016.

28. Hao J, Wang K, Yue Y, Tian T, Xu A, Hao J, Xiao X and He D: Selective expression of S100A11 in lung cancer and its role in regulating proliferation of adenocarcinomas cells. Mol Cell Biochem 359: 323-332, 2012.

29. Wang LN, Tong SW, Hu HD, Ye F, Li SL, Ren H, Zhang DZ, Xiang R and Yang YX: Quantitative proteome analysis of ovarian cancer tissues using a iTRAQ approach. J Cell Biochem 113: 3762-3772, 2012.

30. Sakaguchi M, Murata H, Sonegawa H, Sakaguchi Y, Futami J, Kitazoe M, Yamada H and Huh NH: Truncation of Annexin A1 is a regulatory lever for linking epidermal growth factor signaling with cytosolic phospholipase $\mathrm{A}_{2}$ in normal and malignant squamous epithelial cells. J Biol Chem 282: 35679-35686, 2007.

31. Sakaguchi M, Miyazaki M, Takaishi M, Sakaguchi Y, Makino E, Kataoka N, Yamada H, Namba M and Huh NH: S100C/A11 is a key mediator of $\mathrm{Ca}^{2+}$-induced growth inhibition of human epidermal keratinocytes. J Cell Biol 163: 825-835, 2003.

32. Sakaguchi M, Sonegawa H, Murata H, Kitazoe M, Futami J, Kataoka K, Yamada H and Huh NH: S100A11, an dual mediator for growth regulation of human keratinocytes. Mol Biol Cell 19: 78-85, 2008.

This work is licensed under a Creative Commons Attribution-NonCommercial-NoDerivatives 4.0 International (CC BY-NC-ND 4.0) License. 